\title{
Design of Intelligent Home Planting System Based on Raspberry Pi

\author{
Fengwen Cheng ${ }^{1, a}$, Jie Zhang ${ }^{1, b^{*}}$, Bo Liu ${ }^{1, c}$ and Lei Ye ${ }^{1, d}$ \\ Sichuan Agricultural University, China, 625014, Ya an \\ a979998690@qq.com , b185511591@qq.com ,'3314965794@qq.com,,240683807@qq.com
}

Keywords: Raspberry Pi; Temperature and humidity detection; Automatic Irrigation; Remote monitoring; Ultrasonic distance measurement

\begin{abstract}
Aiming at the problems of high cost, limited function and weak real time interaction of common family planting system, our paper proposed an intelligent family planting system based on raspberry pi. The system is composed of controller, monitor terminal, server and client with the language of Java and python. It can realize data acquisition, automatic level control, real-time monitoring, and it also develops client to provide information interaction for users. Experiments show that the system can realize remote monitoring of home planting through Web browser or APP terminal, and realize real-time data display and automatic supply of nutrient solution, which has good application value.
\end{abstract}

\section{Introduction}

With the development of Internet of Things, the concept of "houseplant factory" has begun to enter people's lives, and the use of soilless cultivation techniques to grow vegetables or flowers on the family balcony has gradually become a trend. Home-growing can not only satisfy people's demand for pollution-free vegetables, but also provide a leisure way ${ }^{[1]}$. Eco-family planting of plant plants uses resource recycling technology, which not only improves resource utilization, but also achieves zero pollution and zero emissions ${ }^{[2]}$.However, in today's households, the use of soilless cultivation techniques for self-cultivation of vegetables has many problems, for example, the inadequacy of care. At the same time, the existing product systems on the market are of high cost and poor scalability ${ }^{[3]}$, and their functions are relatively monotonous that cannot meet the needs of planting enthusiasts. The Raspberry Pi, as an ARM-based micro computer motherboard, has all the basic functions of a PC and is developed by the British Raspberry Pi Foundation. The price is relatively low, and it is highly scalable. Therefore, an intelligent home planting system based on the Raspberry Pi that can realize monitoring and automatic irrigation is a cost-effective implementation solution.

\section{System structure}

The smart home planting system based on raspberry pie is made up of Raspberry Pi server, hardware terminal and client (Web client and Android client). The system structure diagram is shown in Figure 1.The Raspberry Pi server is based on an embedded platform and implements functions of system server design, database setup and background services, and carries out data storage and client polling display. The hardware terminal uses a variety of sensing devices to communicate with the Raspberry Pi through the universal I/O port to realize real-time monitoring of environmental data such as temperature and humidity, and to realize the automatic irrigation function of the nutrient solution. And the system uses the dedicated OV5647 lens of Raspberry Pi to achieve real-time monitoring of the plant environment through the CSI interface ${ }^{[4]}$.The client is based on the network communication technology, using the interface development, data exchange, Android network communication technology to build Web client and Android client, to achieve the visual processing of the collected data in the form of an interface, which can facilitate the user to view the growing environment around the plant. 


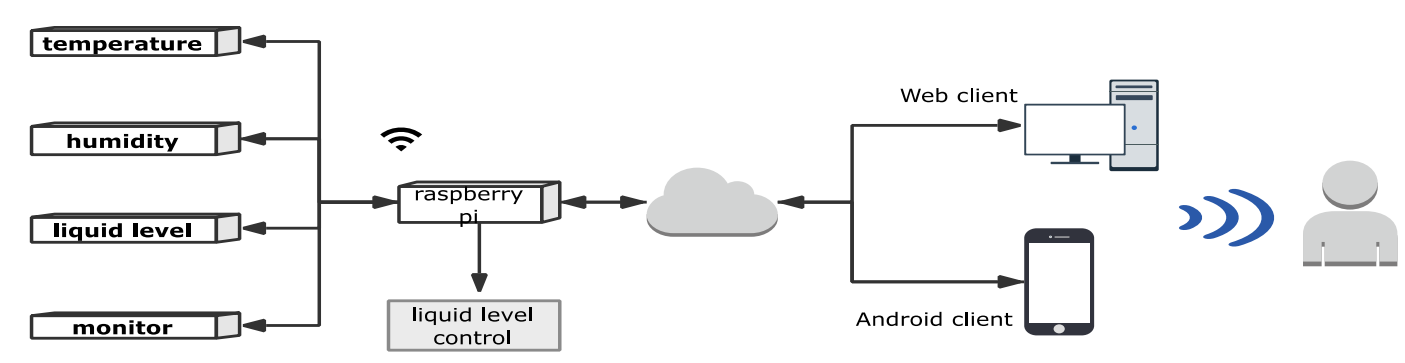

Fig. 1 System structure diagram

\section{Raspberry Pi Server Design}

Building a Raspberry Pi server requires burning the Raspbian image for root directory expansion and providing operating system support, and installing the MySQL database, modifying the system configuration optimization environment, creating data tables, providing data storage services. And also need to install and configure tomcat, peanut shell implementation Service deployment and intranet penetration, provide web client HTTP access support; finally design python daemon to achieve data collection, response control between the terminal and the server.

Build the Raspbian system. This system adopts 3rd generation of B-type development board based on Linux system. It uses SD card as storage medium and supports programming languages such as Python, C, Perl, etc. The system is developed based on Python language. Raspbian is a customized version of Raspberry Pi which is optimized from ARM architecture by Debian Linux. It is also the release version selected by most Raspberry Pi developers which supports ssh login. After establishing a connection, we can directly use Putty to $\log$ in to Raspberry Pi. Then $\log$ in to Raspbian, expand the root directory, perform sudo raspi-config, and select the Expand Filesystem option to expand the Raspbian storage space to its own SD card capacity. Install the remote control daemon xdrp and use the rdp protocol to access the remote Raspberry Pi desktop. The update of the Raspberry Pi's internal software is provided by the foreign mirror station by default, but there are problems such as slow download speed and instability of network. The software source provided by the replacement of the domestic mirror station can largely remedy the above deficiencies. After the source change is completed, perform sudo apt-get update and sudo apt-get upgrade to ensure that the software download package is the latest version.

Database design. MySQL ${ }^{[5]}$ is a relational database that uses the most commonly used SQL language for database management. Compared with large databases such as SQL Server, MySQL can work on different platforms with the advantages of high portability, simple installation and high efficiency, which can effectively reduce the running load of the Raspberry Pi server. And it has a variety of data storage types to meet the storage of data measured by different sensors.

Our system uses MySQL database to build a storage table corresponding to the collected data. When constructing a temperature and humidity data table, three fields of DHT-TIME, DHT-TEMP, and DHT-HUM are used. The timestamp is obtained by the STRFTIME function after formatting it's conversion, and the temperature and humidity are marked with decimal, the variable-length floating point number. Next modify the /etc/mysql/my.cnf file to open the database's remote port for connections between host and client and data access.

Build the Web server. Our system uses peanut shell and tomcat to achieve web server construction. The Tomcat server, as a lightweight web application server, is suitable for small and medium-sized systems and occasions with fewer concurrent access users. Tomcat binds the IP address and listens on the TCP port, and supports running Servlet/JSP applications that can dynamically generate resources and return to the client. Peanut shell has the functions of desktop domain name management and dynamic domain name resolution (DDNS). The mapping method is rich and easy to manage. It is used to realize intranet penetration, so that the Raspberry Pi server can provide external services and achieve client access. 
Background programming. The Raspberry Pi background service program of this system adopts Python as the scripting language, and uses the written program to realize the system's data communication, feedback control and real-time monitoring function.

The data communication collects data through the serial port communication between the DHT11 and the Raspberry Pi, and transmits the data by the wireless sensor network, and displays it through the Web and the Android terminal. The feedback control utilizes the characteristics of the relay switch to perform high and low level conversion under certain conditions to realize automatic replenishment of the nutrient solution; And the real-time monitoring uses the Picam module to monitor the plant's growing environment and provide data browsing services on an interactive web page.

\section{Hardware function design}

The hardware part of the system mainly includes temperature and humidity data acquisition module based on wireless sensor network technology, liquid level detection and nutrient solution automatic supplement module based on HC-SR04, and real-time monitoring module based on Picam.

Temperature and humidity module. The temperature and humidity module is implemented with DHT11 sensors. The DHT11 is a temperature and humidity composite sensor with a calibrated digital signal output ${ }^{[6]}$,including a resistive wetted component and an NTC temperature measuring component. It is small in size and low in power consumption.It is available in 4-pin single-row pin package mode, which is VDD, DATA, NC and GND.

The temperature and humidity measurement module communicates with the Raspberry Pi through a generic GPIO pin to implement data and command interaction. The temperature and humidity sensor can work after being powered on, and output high and low levels to the Raspberry Pi pin after receiving the correct start command.This system uses BCM code to map different temperature and humidity data values. The Raspberry Pi background running program converts the level stream into a digital signal according to a specific coding method, and then converts it into a specific value to realize the collection and upload of the ambient temperature and humidity data.Considering the problem of poor stability of dht11, the background program is used to filter the collected data, and the database operation is performed for the value in the set range, otherwise it is discarded.The flow chart of data collection is shown in Figure 2.

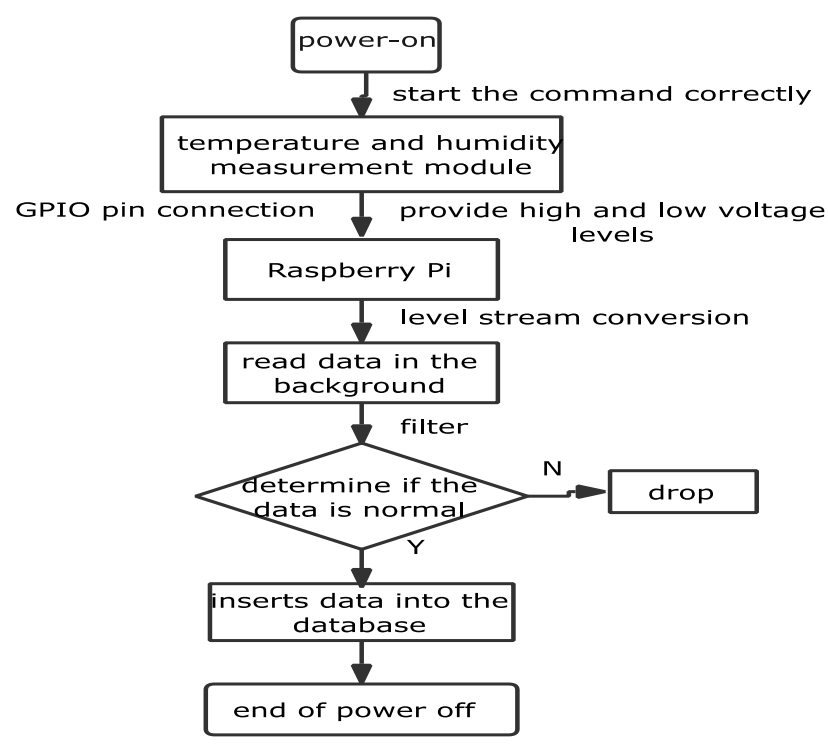

Fig. 2 Data collection flow chart

Liquid level control module. The liquid level control module consists of an ultrasonic distance measuring sensor HC-SR04 ${ }^{[7]}$, a relay and a peristaltic pump.In the design of this system, HC-SR04 is placed on the side of the wall of the liquid container to be tested, and the TRIG port of IO is used for distance measurement.Firstly, a high level signal of $10 \mathrm{us}$ is sent to it, and the module 
automatically sends eight square waves of $40 \mathrm{khz}$ for automatic detection. When there is a signal return, it outputs a high level through the ECHO port of the IO. The duration is the time it takes for the ultrasound to eventually pass back, and the distance between the object to be measured can be obtained by using the sound wave transmission speed.The relay, as an electrical control device in the system design, causes a predetermined step change in the controlled output quantity in the electrical output circuit when the input quantity changes to the specified requirements. It has the interaction between the control system and the controlled system, and is applied to the automatic control circuit, which is essentially an "automatic switch" for small current control of large current operation. The peristaltic pump consists of drive, pump head and hose. It can be powered directly from the Raspberry $\mathrm{Pi}$, enabling pumping of liquids.

In the system design, HC-SR04 is used to return the distance from the liquid surface. Set the maximum and minimum thresholds, and convert the detection of the lack of liquid level and overflow to the distance between the liquid surface and the sensor, and convert the abstract amount to the specific amount. To realize the automatic control function, it is necessary to design a relay circuit module. The relay IN pin is connected to the Raspberry Pi GPIO, the relay output uses a normally closed contact, and the normally closed contact and the common interface are respectively connected to the two metal pieces of the peristaltic pump. The input control signal of the relay is provided by the converted distance. When the ranging is greater than the maximum threshold, a high level signal is generated to cause the relay to open and thereby control the peristaltic pump to operate. When the distance is less than the minimum threshold, a low level signal is generated to turn off the relay. As a result, the nutrient solution can be automatically replenished when the liquid level is lower than the set threshold. The liquid level control flow chart is shown in Figure 3.

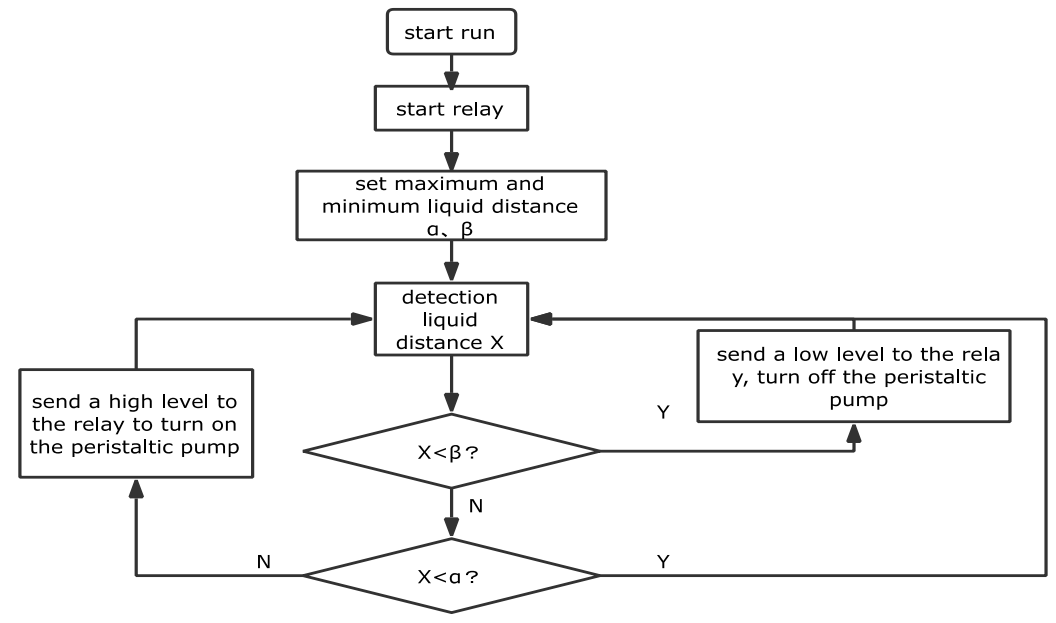

Fig. 3 Flow chart of liquid level control

Real-time monitoring module. As a highly integrated micro motherboard, the Raspberry Pi is equipped with a CSI interface for the camera.Our system selects a dedicated camera to connect with the Raspberry Pi. The specific implementation steps are as follows.

1) Turn on the Enable Camera in the system settings to make it available.

2) Install mjpg-streamer, libjpeg8-dev, cmake and other auxiliary tools.

3) Set the parameters of fps, width, height, etc. in input_raspicam.c in mjpg-streamer according to requirements.

4) compile mjpg-streamer.

5) create a new sh file to run the code, use the chmod744 command to set permissions.

6) Add the command to run the script to /etc/init.d to enable booting from the boot.

7) restart the Raspberry Pi.

After implementing the above steps, the real-time monitoring screen obtained by the Raspberry Pi camera can be accessed through the port 8080 in the browser. 


\section{Client design}

The client consists of a web client and an Android client. It is built using the MVC framework to implement the client synchronization server to monitor data.

Web client design. The Web client adopts the $\mathrm{B} / \mathrm{S}$ structure ${ }^{[8]}$, and the user can implement data interaction with the server through the client. This system uses SpringBoot ${ }^{[9]}+$ Mybatis framework, combining with MySQL database, instantiating MVC frame, to achieve the function of drawing curves and displaying pictures. The SpringBoot framework simplifies the complex configuration of the Spring framework and collects a large number of libraries. Users only need to access the web page to browse the information to generate data interaction.

The server uses Tomcat and peanut shells to implement Web service deployment and intranet penetration.From the software structure division, the implementation of the Web client can be divided into two parts: the Web display page and the back-end data processing. The front-end display page adopts the echart chart, uses Ajax request to dynamically obtain the encapsulated Json data and loads it, and finally displays it in the form of a line chart. The back-end data processing is developed based on Java, implementing Restful API, database access, and Json data formatting.

In the web client, the temperature and humidity data information is converted into a data curve. The real-time data is obtained through the Raspberry Pi serial port and uploaded to the database, and displayed in a graphical form; the image is collected in real time by using the Raspberry Pi terminal. The web client is shown in Figure 4.

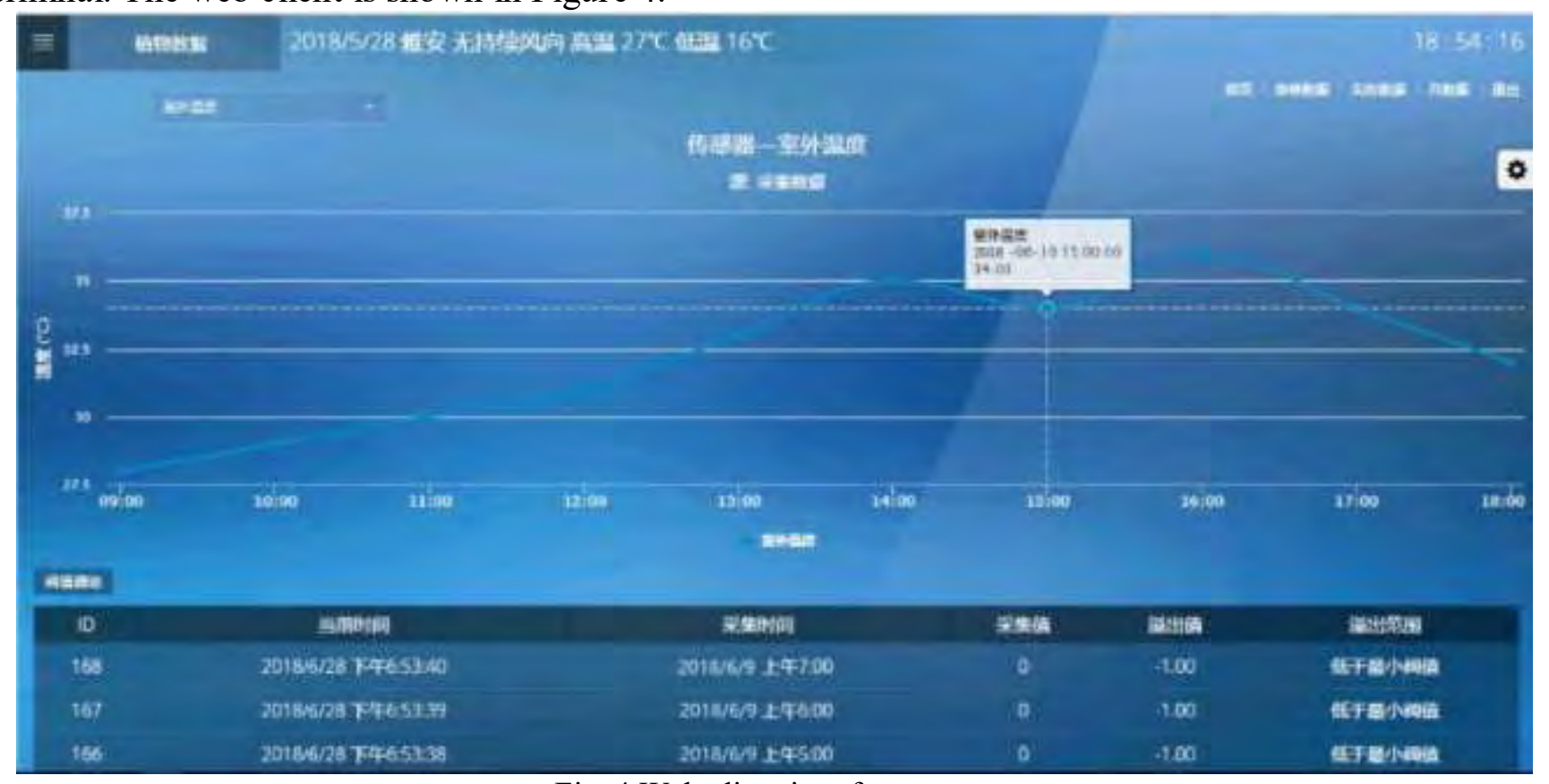

Fig. 4 Web client interface

Android client design. Android can be used in a variety of platforms and applications, and has a promising future ${ }^{[10]}$. The client adopts a mobile device/server mode, and the user can obtain server information anytime and anywhere through the mobile device. The system realizes the graphical display of data on the mobile device, and relies on the TCP/IP network technology to acquire the system data collected by the sensor in real time, and updates the data at regular intervals to ensure the data is valid in real time. The development of the Android terminal adopts a three-tier architecture, namely the UI, BLL and DAL, which can achieve high cohesion, low coupling, and ensure data security.

The Android client implements the function of browsing temperature and humidity data.In the construction of this Android system, the MPAndroidChart chart framework and the OkHttp network communication framework are integrated.MPAndroidChart draws various statistical charts on Android devices and supports dragging and zooming charts. The Android system establishes a TCP connection with the server through OkHttp at regular intervals to obtains the database temperature 
and humidity data, and transmits the data to the MPAndroidChart chart framework for presentation of the presentation layer.

Two different interfaces are designed in the Android client: the main control interface and the monitoring data display interface. The main interface is used to implement user interaction and display optional jumps; the monitoring data display page provides real-time data display in the form of curves and sets alarm thresholds. The main interface and data display interface are shown in Figure 5.

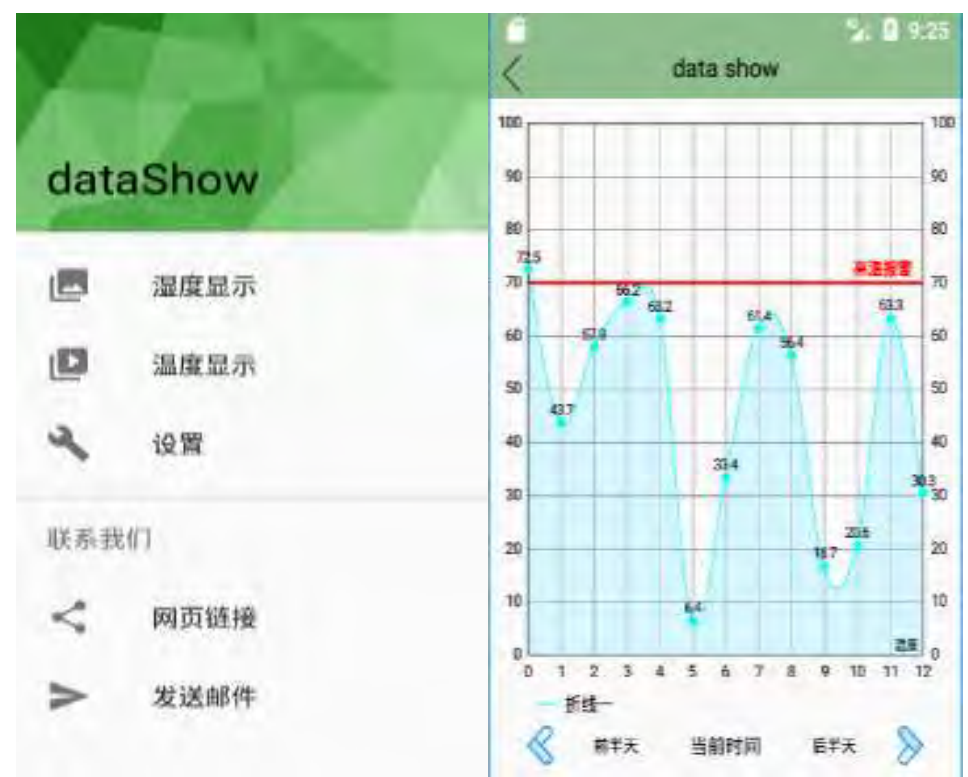

Fig. 5 Android client interface

\section{Conclusion}

This design adopts the 3rd generation B model of Raspberry Pi as the development board of intelligent home planting system, which has the advantages of strong expansion and high cost performance that can reduce the development cost of the ordinary home automatic planting system. And uses ultrasonic distance measuring sensor and relay combination module to achieve automatic irrigation, instead of the ordinary manual control valve watering method, which is more intelligent.

The system can provide intelligent home planting service function, and users can access the plant growth environment in the home through real-time monitoring through the smart mobile device or browser webpage, and realize intelligent automatic irrigation. The system works stably and reliably, has good scalability, and has great practical value.

\section{References}

[1] Q.Q.ZHANG, Research on Aeroponics-based Family Plant Factory Design and Spraying Frequency Optimization[D].Zhejiang: Zhejiang University,(2017). In Chinese

[2] Y.Y.LI. Research and Design on Household Planting Device System.Shanghai: Donghua University,(2014). In Chinese

[3] X.X.GU,N.L.Ning and C.Y.ZHU. Application status, problems and Countermeasures of family plant factories[J].Agricultural Engineering Technology, Vol.36, (2016) p.36-39. In Chinese

[4] X.H.YIN,M.Y.LIU and N.WANG. Design of smart home system based on Raspberry $\mathrm{Pi}[\mathrm{J}]$.Modern electronic technology, Vol.23,( 2016) p.161-164. In Chinese

[5] C.Y.GONG ,J.H.XING and Y.B.HU.Do communication of Android mobile terminal and PHP and MySQL based on JSON[J].Industrial instruments and automation devices, (2013) No.1 p.63-65. In Chinese 
[6] Z.H.WANG and C.Z.BAI .Design and implementationof multi-point temperature and humidity alarm systeminlaboratory based on DHT11[J].Shanxi Electronic Technology, (2010) No.6, p.60-62.

[7] X.P.TANG and X.S.XIE. Design of the opposite-type ultrasonic ranging system based on HC-SR04 integrated module[J].Microcomputer and application, (2014) No.22,P.90-93. In Chinese

[8] W.K.WANG and L.YAN. System Design of Intelligent Greenhouse Based on B/S Structure and Open Source Yeelink Platform[J].Electronic Technology and Software Engineering,(2017) No.8,p.169-170. In Chinese

[9] F.ZHANG, Applying SpringBoot to Change the Web Application Development Model[J].Science and Technology Innovation and Application[J].Technology Innovation,(2017) No.23, p.193-194. In Chinese 